Artículo de investigación resultado de la "Estrategia integral del control a las rentas ilícitas para el fortalecimiento de las rentas oficiales como fuente de inversión social en el departamento de Antioquia" a través del convenio interinstitucional entre la Secretaría de Hacienda de la Gobernación de Antioquia y la Institución Universitaria Tecnológico de Antioquia, Medellín - Colombia, 2018.

\title{
Los fraudes en las rentas departamentales: una mirada desde la institucionalidad a las dimensiones social, económica, legal y normativa
}

\author{
The frauds in the departmental rents: A look from the institutionality to the social, \\ economic, legal and normative dimensions
}

Recibido: 03-03-2019 • Aprobado: 29-05-2019 • Página inicial: 213 - Página final: 229

\author{
Luisa Fernanda Zapata Álvarez* \\ Leidy Jhojana Usma Zuleta**
}

\begin{abstract}
Resumen: este artículo emerge como resultado del proceso de investigación que ha tenido lugar en el convenio entre la Secretaría de Hacienda del Departamento de Antioquia y el Tecnológico de Antioquia - Institución Universitaria. U., en el marco de la Estrategia Integral al Control de las rentas ilícitas. En el texto se desarrollan algunas afectaciones en las dimensiones social, económica, legal y normativa a partir de la ocurrencia de fraudes en cinco rentas en el departamento de Antioquia. Es una investigación cualitativa con método hermenéutico, que utilizó fuentes escritas y orales a través de la revisión documental, la encuesta y la entrevista semiestructurada a personal relacionado con las rentas departamentales en los distintos municipios de Antioquia. El análisis se realizó a partir de un proceso de sistematización, categorización y triangulación de la información, y dio como resultado las categorías relacionadas en cada una de las dimensiones y analizadas a la luz de la cultura de la legalidad y las buenas prácticas.
\end{abstract}

Palabras clave: dimensión social, dimensión económica, dimensión legal y normativa, fraude, buenas prácticas, cultura de la legalidad.

\begin{abstract}
This article emerges as a result of the research process that has taken place as part of the agreement between Department of Antioquia' Tax and Public Finance Secretariat and the Tecnológico de Antioquia - I.U., within the framework of the Comprehensive Strategy to Control illicit income. The text develops some effects on the social, economic, legal and regulatory dimensions from the occurrence of fraud in fi ve rents in the department of Antioquia. It is a qualitative research with a hermeneutical method that used written and oral sources through the documentary review, survey and semi-structured interview with personnel related to the departmental rents in the different municipalities of Antioquia. The analysis was made from a process of systematization, categorization, and triangulation of information, resulting in the categories related to each of the dimensions and analyzed in light of the culture of legality and good practices.
\end{abstract}

Keywords: Social dimension, economic dimension, legal and regulatory dimension, fraud, good practices, culture of legality.

JEL: E01

\footnotetext{
* Psicóloga de la Universidad de Antioquia, Especialista en Psicología Social Aplicada y Magister en Psicología Social de la Universidad Pontificia Bolivariana. Investigadora del Grupo de Investigación Observatorio Público, equipo de sistematización de la Estrategia Integral del Control a las Rentas Ilícitas del Tecnológico de Antioquia, Medellín - Colombia. luisa.fza@gmail.com - ORCID: http://orcid.org/0000-0002-3659-6446

** Contadora Pública del Politécnico Colombiano Jaime Isaza Cadavid. Investigadora del Grupo de Investigación Observatorio Público, equipo de sistematización de la Estrategia Integral del Control a las Rentas Ilícitas del Tecnológico de Antioquia, Medellín - Colombia.

jhojausma@hotmail.com - ORCID: http://orcid.org/0000-0002-5824-9824
} 


\title{
Contexto zapata L Lysman L
}

\section{Fraude no rendimento departamental: Um olhar da institucionalidade para dimensões sociais, econômicas, legais e regulatórias}

\begin{abstract}
Resumo: este artigo surge como resultado do processo de pesquisa que ocorreu no acordo entre a Secretaria do Tesouro do Departamento de Antioquia e o Tecnológico de Antioquia - Instituição Universidade U., no âmbito da Estratégia Integral ao controle de renda ilegal. No texto algumas afetações se desenvolvem em social, econômico, jurídico e regulamentos da ocorrência de fraude em cinco aluguéis no departamento de Antioquia. É uma pesquisa qualitativa com método hermenêutica, que usava fontes escritas e oral através da revisão documental, a pesquisa e a entrevista semiestruturada ao pessoal relacionado à renda departamental nos diferentes municípios de Antioquia. A análise foi feita a partir de um processo de sistematização, categorização triangulação de informações e deu como resultar nas categorias relacionadas em cada uma das dimensões e analisada à luz da a cultura da legalidade e boas práticas.
\end{abstract}

Palavras-chave: dimensão social, dimensão económica, dimensão jurídica e regulamentos, fraude, boas práticas, cultura da legalidade. 


\section{Introducción}

La Gobernación de Antioquia en el periodo 2016-2019 implementó varias estrategias encaminadas al desarrollo social, económico y cultural del departamento, con el propósito de fortalecer las comunidades y la institucionalidad. Estos esfuerzos por el fortalecimiento de las comunidades deben ser para las administraciones públicas un aspecto fundamental, pues en la construcción e implementación de prácticas de buen gobierno se pueden mejorar diferentes esferas de la sociedad. Ningún gobierno puede relativizar su responsabilidad cuando se trata de cumplir misiones de servicio público, incluso si éstas se encomiendan al sector privado (Figueroa y Orellana, 2007, p.170).

Existen desarrollos que sugieren que la institucionalidad pública es también dependiente de la medida en que los valores de la solidaridad, conciencia cívica y dedicación humana se asienten en la producción de los servicios sociales. Esto, no sólo porque a través de ellos los servicios tienen más probabilidades de maximizar su eficiencia (en un sentido amplio) y su equidad, sino porque la intención expresa de su fortalecimiento puede contribuir a reforzar la dimensión de deberes de la ciudadanía y, específicamente, a desarrollar capacidades y organizaciones sociales (Cunill, 2010, p.65).

Ahora bien, "La Estrategia Integral de control a las rentas ilícitas para el fortalecimiento de la inversión social en el departamento de Antioquia" (en adelante Estrategia Integral), es uno de los grandes esfuerzos de la Gobernación para hacerle frente a la ilegalidad y para proponer buenas prácticas en torno a una cultura de legalidad. Dentro de su concepción se establecen varios componentes para su desarrollo: comunicación, tributario, judicial y participativo. La Estrategia se concentra en cinco rentas específicas: monopolio rentístico de alcoholes potables y licores destilados, monopolio rentístico de juegos de suerte y azar, impuesto al consumo de cigarrillos y tabaco, impuesto de degüello, y sobretasa a la gasolina (Usma y Zapata, 2018, p.431), y se actúa según la particularidad de cada una.

Desde su inicio, además, se consideraron de gran importancia los ejercicios académicos e investigativos dentro de ella, y se estimó que dejar plasmada una sistematización de experiencias, análisis de impacto y reflexiones alrededor de la cultura de la legalidad y las buenas prácticas, contribuiría a fortalecer la relación Universidad-Estado-Sociedad. 


\section{Contexto zaosal L L y usna, L}

El Tecnológico de Antioquia pone de manifiesto la importancia de interrelacionar su discurso académico con las necesidades más apremiantes de las comunidades humanas que hacen parte de su quehacer, en este caso, las poblaciones hacia las cuales está dirigida la estrategia gubernamental de rentas (Gómez, Usma, Zapata, Echavarría y Giraldo, 2017, p.37).

De dichos ejercicios se pueden resaltar varios productos de investigación, en los cuales se han abordado temas que reúnen la cultura de la legalidad, los fraudes y los riesgos asociados a cada una de las rentas que aborda la estrategia, así como buenas prácticas comunitarias e institucionales y análisis de impacto. En este sentido, en la investigación realizada sobre buenas prácticas de legalidad e instituciones responsables, se tuvo como objetivo general caracterizar las buenas prácticas en las rentas departamentales desde las instituciones de la Gobernación de Antioquia y se trazaron los objetivos específicos: a) identificar el conocimiento que tienen los funcionarios departamentales sobre la cultura de la legalidad en el marco de la Estrategia Integral; b) describir las acciones que conocen los funcionarios departamentales que contribuyen al fortalecimiento de la cultura de la legalidad, y c) sistematizar las recomendaciones que tienen los funcionarios en materia de buenas prácticas. De allí se desprende el presente artículo que aborda lo social, lo económico y lo legal y normativo, como dimensiones en las cuales los fraudes en las rentas tienen incidencia y afectaciones que comprometen el resultado de la Estrategia Integral.

La metodología que se utilizó para la investigación es de tipo cualitativo con un método hermenéutico, a través del uso de la encuesta, la entrevista semiestructurada y la revisión documental, y se dio paso a un proceso de sistematización y análisis de la información. Los resultados y la discusión se plantean a partir de las tres dimensiones anteriormente mencionadas (lo social, lo económico y lo legal y normativo) que aportan temas relevantes que se analizan a la luz de la cultura de la legalidad y las buenas prácticas institucionales.

\section{Metodología}

La presente investigación se fundamenta desde el paradigma cualitativo, considerando que los objetivos trazados apuntan, precisamente, a la comprensión de la realidad sobre los fraudes y las buenas prácticas en las rentas departamentales a partir el discurso, la práctica y el contexto de los propios participantes y reconociendo los significados que construyen en su propia realidad. De esta manera, en esta investigación se reconoce que "el 
conocimiento es un producto social y su proceso de producción colectivo está atravesado e influenciado por los valores, percepciones y significados de los sujetos que lo construyen" (Galeano, 2013, p.18). Como el análisis se realiza desde la dimensión interpretativa de la producción oral y escrita de los participantes, se utiliza el método hermenéutico, el cual, en palabras de Habermas (2000, citado en Rojas, 2011, p.187) "es un recurso metodológico de la ciencia social, que aborda en el medio que es el lenguaje en funciones (de socialización, de reproducción cultural y de integración social) el análisis de expresiones de significados, de sentido, de tales expresiones".

Para el desarrollo de la investigación se tuvieron en cuenta dos tipos de fuentes, por un lado las fuentes orales que "aportan su mirada al problema que se trata de comprender" (Galeano, 2013, p.44) y, por otro, las fuentes escritas que permitieron "complementar y confrontar las fuentes orales" (Galeano, 2013, p.45). De acuerdo a los objetivos y características propias de la investigación se utilizaron la revisión documental, la entrevista semiestrucurada y la encuesta como técnicas de recolección de información, haciendo uso de instrumentos como guías de entrevistas, cuestionarios y fichas de recolección biliográfica.

\section{Sistematización y análisis de la información}

Luego de transcribir y organizar la información que arrojaron las entrevistas, la encuesta y la revisión documental, se pasó a un proceso de sistematizar, codificar y categorizar la información, que permitió "caracterizar y clasificar datos, permitiendo su vinculación con la teoría (...) que permiten analizar los datos existentes, establecer relaciones entre ellos y presentarlos de manera clara y concreta" (Galeano, 2013, p.46). Se utilizaron matrices de coherencia y matrices intertextuales que posibilitaron realizar un proceso de triangulación y análisis de la información, mediante el cual la información recogida a través de los instrumentos se puso en relación con los conceptos propios de las buenas prácticas y la cultura de la legalidad.

\section{Resultados}

En el Departamento de Antioquia se evidencian fraudes asociados con las rentas que aborda la Estrategia Integral, que tienen una afectación en diferentes dimensiones: social, económica, legal y normativa. La importancia de estas características no recae sobre una dimensión en específico porque se considera que ellas van relacionadas entre sí; la afectación de una de ellas inmediatamente involucra los beneficios de las otras. Por lo tanto, se presenta una relación entre 


\section{Contexto zapata L L y ysma, L}

la descripción de cada una de las dimensiones con unas categorías que se van desarrollando en el texto.

A continuación, se presentan las tres dimensiones en las cuales se identifican afectaciones y se brindan al lector elementos para identificar cada una de las facetas en las que se desenvuelve la Estrategia Integral para el control de las rentas ilícitas, lo que permite generar comprensión y reflexión sobre la cultura de la legalidad que desembocan en la construcción de prácticas de sensibilización, transparencia y respeto entre los diferentes actores de la sociedad (instituciones, comerciantes, distribuidores y comunidad).

\section{Dimensión social: dinámicas sociales, comunitarias, y vecinales}

Se puede decir que esta dimensión reúne a casi todos los actores de la sociedad, sus dinámicas culturales y de relacionamiento son construidas a lo largo del tiempo y muchas se han repensado y mejorado en la actualidad. Sin embargo, a lo largo de la historia Antioquia ha presentado prácticas de ilegalidad respecto de las rentas $y$, por lo tanto, hoy es una tarea inacabada pero que se fortalece, prueba de ello es la estrategia a la que se está haciendo referencia en la presente investigación.

Por lo mismo, la estrategia propone prácticas que generan un impacto significativo en la dimensión social y crean, en torno a las comunidades, dinámicas que transfiguran identidades colectivas. Se trata de "la necesidad de hacer una mirada a las dificultades que se presentan en la sociedad actual en el ámbito de la construcción de comunidad, entendida como la relación con otros que son nuestro prójimo, nuestro próximo" (Bauman, 2005, p.67).

Cuando se habla de dimensión social se hace referencia al espacio y el contexto en los cuales las acciones repercuten en el desarrollo de la sociedad. Es decir, los fraudes tienen un impacto completo por el espacio y el tiempo que abarcan, y llegan a ser complejos a causa de las identidades individuales. Cuando se plantean formas de relación solidarias, se revelan cansancio e incredulidad por parte de la comunidad (Téllez, 2010, p.17), porque la ilegalidad ha estado latente por generaciones en el desarrollo del histórico colectivo del departamento de Antioquia. Pero a partir de unas buenas prácticas se pueden generar "formas de relación denominadas democráticas, justas, equitativas, respetuosas, plenas de apoyo y solidaridad, es preciso darles otro sentido desde la práctica misma para que se llenen de contenido vital para las comunidades" (Téllez, 2010, p.17). Con las buenas prácticas se debe intentar contrarrestar los flagelos que se 
presentan en esta dimensión para generar, además, importantes acercamientos y trabajos colectivos.

El cuidado da la posibilidad de tener perspectivas más amplias de empatía con el otro en su reconocimiento; de disponerse a escuchar de manera atenta entendiendo las necesidades e intereses de ese otro; de ponerse en el lugar del otro, y de enriquecerse al reconocer ser distintos y plurales (Téllez, 2010, p.20).

En este sentido, los participantes de la investigación resaltan dos elementos significativos anudados a la dimensión social en función de la afectación de los fraudes sobre la misma: las particularidades del contexto y las prácticas sociales de los territorios.

\section{En contexto, la particularidad de las prácticas sociales}

Para los participantes de la investigación las afectaciones que tienen los fraudes que se cometen en las rentas que se han mencionado, están relacionados directamente con las particularidades de cada contexto. En la investigación que se desarrolló se pudo conocer que aunque las distintas subregiones presenten problemáticas similares, es decir, aunque vivencien los mismos fraudes, estos se pueden significar y practicar de manera distinta y a su vez se encuentren afectaciones diferentes en los territorios y pobladores. Los participantes distinguen desde las zonas en las que se encuentran -urbana, rural, ribereña, costera- hasta las economías legales que las atraviesan -minería, manufactura, agroindustria, turismo, etc.--

Es así como las afectaciones de los fraudes en la dimensión social están atravesadas por las diferenciaciones geográficas, económicas, territoriales y culturales, lo cual implica que sea necesario reconocerlas y comprenderlas para el ejercicio de buenas prácticas institucionales en el marco de la estrategia desde sus distintos componentes.

En la misma vía, los participantes advierten asuntos con relación a las prácticas sociales que se construyen en cada territorio, que no solo se diferencian entre las subregiones, sino, incluso, en los municipios de las subregiones. En este caso, se manifiesta que las afectaciones de los fraudes pasan por la construcción de sentido de comunidad, de pertenencia y de reconocimiento de la historia social e identitaria que los une como sujetos sociales y en relación (Montero, 2007), y que, en este marco, no solamente se dan los fraudes como construcciones 


\section{Contexto zapaa L L y ysma L L}

colectivas de ilegalidad, sino que también se pueden presentar mecanismos para afrontarlos y buenas prácticas institucionales y comunitarias.

En este sentido, con esta clase de comprensiones el ciudadano puede empezar a identificar que el fortalecimiento de las rentas departamentales, más allá de aumentar la destinación para proyectos de inversión social, genera construcciones locales de tolerancia y respeto por el otro, estimula el trabajo mancomunado con las instituciones e incentiva la participación ciudadana, por eso en esta dimensión se generan dinámicas sociales, comunitarias y vecinales en pro del desarrollo.

\section{Dinámicas sociales, comunitarias y vecinales}

La dinámica social, como ya se abordó, engloba las áreas del constante cambio en la sociedad. "La dinámica social se encarga de evaluar y analizar el cambio y evolución de los modos de producción, las organizaciones e instituciones sociales y su interacción con la realidad social, económica, cultural, política, ecológica, etc.” (Incorporación, inclusión e integración, 2009, párr.1).

Por otro lado, aunque la afectación desde la dinámica comunitaria deviene de las dinámicas sociales, se encuentra situada en una comunidad particular.

La comunidad debe ser entendida más allá de lo geográfico, pues se crea y se recrea más allá de los límites del espacio; es dinámica, toma formas distintas dependiendo de su entorno externo e interno; se adapta, cambia, nunca da respuestas iguales; se regula a partir de sus propias condiciones. (Téllez, 2010, p.14).

La organización comunitaria es participativa, mejora la vida de sus comunidades, es un proceso "por el cual la gente se agrupa para identificar problemas o metas comunes, para movilizar recursos $\mathrm{y}$, de otras formas, desarrollar e implementar estrategias para alcanzar los objetivos que quiere lograr" (Nagy, s.f.).

Mediante la participación el entorno se transforma, dejando impronta e incorporándose en los procesos cognitivos y afectivos de manera activa. Y a la inversa, a través de la identificación simbólica, el espacio apropiado pasa a ser un factor de continuidad y estabilidad del ser, a la vez que un factor de estabilidad de la identidad y la cohesión del grupo (Vidal, en prensa). Desde esta perspectiva los procesos psicosociales se enmarcan en fenómenos colectivos como la identidad comunitaria o el sentido de comunidad (Berroeta y Rodríguez, 2010, p.9). 
En este sentido, los participantes proponen que las prácticas sociales son importantes no solamente para entender el fraude, sino para comprender sus afectaciones y construir los mecanismos para afrontarlas, a partir de acciones que pasen por la legitimación de las prácticas institucionales, legales y normativas, así como de construcciones comunitarias, colectivas y/o vecinales. Las dinámicas vecinales son aun más particulares que las dinámicas comunitarias y abarcan de manera significativa la convivencia y la solidaridad, se desarrollan en relaciones más estrechas que impulsan al diálogo y a la creación de espacios de responsabilidad social. Estas dinámicas generan empoderamiento entre "los habitantes de los barrios para que tomen decisiones y se comprometan en su cumplimiento a largo plazo, es una de las vías más eficaces para desactivar potenciales focos de conflicto" (Piedra, 2017, p.6).

Los procesos de comunicación, en su versión más humana que es el diálogo, o en sus proyecciones organizativas y mediáticas, son un instrumento de apoyo a las transformaciones en las que el hombre es el centro. No hay desarrollo económico, social o político que no tenga como centro al hombre (Gumucio, 2004, p.8).

Los fraudes en las rentas departamentales impactan dinámicas individuales, vecinales, comunitarias y sociales. Todas están relacionadas entre sí, y la afectación, más allá de los ingresos departamentales, se concentra en la deconstrucción y retroceso de la cultura colectiva antioqueña. De tal manera que el engranaje de relaciones que se reúnen en la dimensión social, y que se pueden particularizar en Antioquia a partir de lo comunitario y lo vecinal, permitan aterrizar tres temas que se particularizaron en los participantes: la inversión social, la cultura tributaria y las afectaciones en salud.

\section{De lo social a lo particular: realidades concretas}

Los participantes de la investigación ponen en evidencia realidades concretas de las afectaciones de los fraudes en la dimensión social. Un elemento importante está relacionado con cómo la ilegalidad deja unas consecuencias directas en la inversión social, esto se traduce en las dificultades que existen, en muchos casos, para desarrollar planes y proyectos en relación con lo social e incluso en infraestructura, considerando que los recursos de las rentas recaudadas se invierten en la salud, la educación e incluso en programas que puedan fortalecer el bienestar social de los pobladores. En este sentido, hay una afectación de orden estructural en asuntos económicos y sociales, pero que se configura en consecuencias concretas, reales y materiales en el acceso de la población a estos programas y proyectos. 


\section{Contexto zapaat Ly ysma L L}

Con respecto a lo anterior, se tiene la cultura tributaria, que trasciende el hecho de las prácticas de ilegalidad como mecanismos aislados de los sujetos, y les da lugar a las construcciones sociales en los distintos territorios sobre el deber ser frente a lo tributario. En algunas comunidades y poblaciones se desconoce esta responsabilidad como ciudadanos y en otros casos no se ha afianzado como una tradición en términos de la cotidianidad y responsabilidad. De ahí que una afectación de los fraudes es la cultura de la ilegalidad que se teje en las comunidades, y se hace un llamado imperativo a dar la vuelta hacia buenas prácticas institucionales y comunitarias que construyan no solamente un hábito de pago de tributo, sino toda una cultura de legalidad con relación a él. Es así como

... se reconoce a la comunidad como un actor social que puede incidir en su propia realidad, transformándola de cara a un mejoramiento en su calidad de vida, en este sentido, y con relación a las rentas del Departamento de Antioquia, las comunidades existentes en este territorio tienen las herramientas para identificar las problemáticas asociadas a las rentas ilícitas y también pueden contribuir a su solución, por lo tanto pueden aportar a las dinámicas que ha propuesto la institucionalidad apropiándose de ellas e incluyéndolas en sus acciones cotidianas para construir una cultura de la legalidad (Usma y Zapata, 2018, p.440).

De esta manera, los participantes advierten que estos escenarios fraudulentos se podrían combatir estructuralmente y no coyunturalmente, y se reducirían las dificultades en inversión social que se mencionaron arriba y las afectaciones en asuntos tan complejos como la salud, que se ven comprometidos por las prácticas fraudulentas.

\section{Dimensión económica: Afectación en los ingresos familiares y regionales}

Esta dimensión abarca el impacto que los fraudes generan en términos de economía y se identifica a través de las afectaciones monetarias que puedan traer al Departamento de Antioquia. Se reconoce, además, que esas ilegalidades traen consigo otras particularidades que involucran aspectos sociales y hasta culturales.

Los impactos económicos pueden ser de corto, mediano o largo plazo, en la medida que (sic) algunos afectan de manera directa la demanda o la oferta del mercado, o modifican variables estructurales en los beneficiarios, que redundan en calidad de vida y productividad en un período mayor. Un ejemplo de impacto en el corto plazo son 
las transferencias en efectivo y el aumento de demanda de bienes de consumo que generan, particularmente de aquellos que componen la canasta básica del hogar. Impactos a mediano plazo son los que se producen por ejemplo con el incremento de demanda por viviendas o matrícula educacional que generan los bonos o subsidios respectivos, los que, al menos al iniciarse los programas, requieren de algunos meses de maduración hasta que se consolida un sector capaz de expandir la oferta para cubrir la demanda incremental. En tanto, el impacto en productividad que genera la universalización de la educación básica y media, necesita de varios años para que se reflejen adecuadamente en los indicadores económicos (Martínez, Palma, Flores y Paz, 2012, p.20).

En esta vía y en relación con la cultura tributaria, los participantes advierten que hay una afectación económica en la baja captación de tributos, con los cuales se financian los gastos públicos y se invierte en desarrollo social. El poco recaudo de impuestos afecta directamente estas financiaciones e inversiones. En este sentido, por ejemplo, que un municipio cuente con pocos establecimientos abiertos al público significa poco recaudo y por ende menor inversión social en el mismo municipio.

La dimensión económica posee una afectación en los ámbitos familiar, vecinal, comunitario, regional y social, que se categorizan de manera conjunta, y se le da gran importancia al hecho de que las rentas departamentales tienen como destinación áreas como la salud, la educación, los deportes y la cultura, las cuales abarcan a todos los actores de la sociedad.

El ingreso familiar se conforma por el total de las ganancias que tiene una familia en común y con él se deben suplir las necesidades básicas del grupo. $\mathrm{Su}$ principal fuente es el empleo y es afectado por diferentes elementos, sin embargo, en este caso su afectación se relaciona con los fraudes rentísticos. Si disminuyera la ilegalidad se podría estimar que la demanda de los productos y servicios legales aumentaría y, por lo tanto, el nivel de producción de los elementos que se relacionan con las rentas de la Estrategia Integral. Si esa aseveración se toma como posibilidad se podría decir que "al aumentar la productividad, es posible pagar mejor a los trabajadores sin generar inflación ni recortar las ganancias de la empresa. De esa forma, el aumento de los salarios puede mantenerse a largo plazo" (Polaski, 2003, p.11). 


\section{Contexto zapata L. Lysma L}

\section{¿La región en desarrollo?}

Ahora bien, la afectación para los ingresos regionales se representa desde un aspecto macroeconómico, pues el fortalecimiento de las rentas departamentales posibilita un crecimiento económico que conviene a los habitantes del departamento. Se requiere una inversión dinámica y creciente para lograr mayores tasas de crecimiento (Revista Semana, 2000, parr.11). Y más allá de recaer en la institución la responsabilidad de fortalecer los tributos, debe compartirse con el mundo empresarial. Es decir, si el ingreso de las rentas disminuye también disminuye el crecimiento, por tanto tiene consecuencias no solo de orden cuantitativo, sino también cualitativo en términos de desarrollo social, en gran parte por la falta de inversión social. Así que es importante erradicar las prácticas de ilegalidad para que se pueda

(...) conformar una visión compartida de país que valide la empresa como unidad básica de generación de riqueza; donde empresarios, trabajadores, gobierno y otros actores sociales se vean beneficiados por el diálogo y el trabajo en equipo, enmarcados en un ambiente de justicia, reglas claras y seguridad, para un desarrollo sostenible (Revista Semana, 2000, parr.16).

De tal manera que los participantes afirman que una de las afectaciones de los fraudes en las rentas es el detrimento económico, que hace referencia al daño a los sectores productivos en los territorios, tales como comerciantes, distribuidores y consumidores y a la comunidad en general.

Así, el afrontamiento en la dimensión económica va más allá del recaudo, y este debe ir ligado a las funciones que necesita la dimensión social, las cuales se van correlacionando y el trabajo en cada una de ellas no debe menospreciar la otra dimensión. Es así como los participantes nombran de qué manera las afectaciones económicas tienen relación con el crecimiento económico y el desarrollo social de la región, es decir que no solamente se afecta el recurso económico en términos de la producción de bienes y servicios, sino también en términos cualitativos en cuanto a la calidad de vida y el bienestar social, ya que para los participantes el desarrollo está relacionado con los procesos de transformaciones cualitativas que se materializan en planes y proyectos de inversión social. 


\section{Dimensión legal y normativa: incumplimiento de la disposición normativa de la región y la nación}

El impacto en la dimensión legal y normativa se evidencia en el incumplimiento de las leyes, el aumento de la ilegalidad y el control fiscal. La afectación negativa al importe de los ingresos departamentales ocurre por las acciones derivadas de la ilegalidad, mejor conocidas con el término de fraudes, las cuales son impulsadas por intereses económicos (Gómez et al., 2017, p.79).

Si bien el incumplimiento de las leyes se puede dar por su desconocimiento, esto no exonera a los ciudadanos de las responsabilidades adquiridas en relación con las rentas departamentales. Ser distribuidor, comerciante o consumidor que evade los impuestos afecta de manera significativa las dimensiones social, económica y legal, pues el dinero que no ingresa al Departamento como concepto de renta significa menor inversión social para la comunidad. Es decir, incumplir la ley significa afectar la dimensión económica, por la disminución de los ingresos, la dimensión social, porque la inversión en los sectores que mejoran el desarrollo se ve afectada de manera negativa, y la dimensión legal, por el incumplimiento de las disposiciones establecidas por la ley.

Las acciones de la comunidad transgreden esta dimensión y las malas prácticas inciden, además, en el desarrollo de la dinámica social y la dinámica económica, "por lo tanto, el nivel de cumplimiento y aceptación se verá afectado por la intervención de la institucionalidad" (Usma y Zapata, 2018, p.438). En el proceso investigativo adelantado en el marco de la Estrategia Integral se han propuesto vías para el mejoramiento a través del fortalecimiento de la cultura de la legalidad y que se traduce en buenas prácticas.

(...) las obras reflejadas en su territorio, la atención social recibida y la inclusión, por lo tanto, el nivel de cumplimiento y aceptación se verá afectado por la intervención de la institucionalidad. Luego de tal aceptación, se podrán identificar cambios a partir del ejercicio y la creación de nuevas y buenas prácticas, no solo pensadas desde la cultura de la legalidad, sino también desde el ámbito comunitario (Usma y Zapata, 2018, p.438).

Esas prácticas de los ciudadanos en el desarrollo de cada una de las rentas se basan en la desconfianza por la institución. Por eso, mientras no se encuentre la forma de crear una "relación sana entre quien rinde cuentas y quien las pide comprende una valoración intrínseca de confianza, es insistir en una 


\section{Contexto zapaal Ly ysman L}

relación entre la institucionalidad y la ciudadanía, en una idea de construir conjuntamente cuestionamientos y consensos respetados en el tiempo" (Usma y Zapata, 2017, p.246).

\section{Conclusiones}

Durante el proceso de investigación en el marco de la Estrategia Integral se han abordado dos categorías centrales como forma de analizar las acciones realizadas en los distintos componentes; se tiene la cultura de la legalidad, entendida como

(...) una construcción que realizan los diferentes actores de la sociedad para regularse a través del cumplimiento efectivo de las normas que se han reglamentado o que por medio de acciones individuales y colectivas se van legitimando e institucionalizando como prácticas adecuadas frente a una situación en particular, para nuestro caso, las diferentes rentas que tiene el Departamento (Gómez et al., 2017, p.42).

En este sentido, comprender las dinámicas que reconocen los actores en la realidad de los distintos municipios de Antioquia frente a los fraudes y con relación a las dimensiones social, económica y legal y normativa, implica reconocer elementos que aporten a la construcción de una cultura de la legalidad, no solo desde vías que impliquen mecanismos de control, fiscalización y judicialización, sino que además posibiliten construcciones colectivas que aporten al desarrollo en términos sociales y económicos desde el análisis estructural de las problemáticas. De esta manera, temas como la cultura tributaria, las prácticas sociales y las particularidades del contexto permiten crear mecanismos y estrategias de transformación social que inciden en combatir la ilegalidad.

En consideración a lo anterior, las buenas prácticas institucionales y comunitarias posibilitan esos escenarios de generación y consolidación de una cultura de la legalidad, teniendo en cuenta que estas prácticas permiten contrarrestar esos efectos o afectaciones que dejan los fraudes. La buena práctica de diferenciación, entendida como "las acciones en materia de buenas prácticas [que] están situadas y contextualizadas en la realidad socio-cultural, económica, histórica y política de las regiones y/o comunidades antioqueñas" (Usma y Zapata, 2017, p.270), apunta, entonces, a tener en cuenta las prácticas 
y acciones sociales con relación a la legalidad no solo desde las afectaciones, sino desde sus significados, lo que contribuye a comprender la afectación en la dimensión social, pero con efectos en las dimensiones económica y legal y normativa.

Las buenas prácticas de innovación y de incidencia pública y social, por su parte, permiten abordar los fraudes y sus afectaciones, en relación, incluso con la diferenciación, es decir, desde acciones situadas y contextualizadas que sean creativas y novedosas y que permitan una construcción colectiva, en esta vía se podría consolidar, como se dijo anteriormente, una cultura de la legalidad que fortalezca las acciones ciudadanas e institucionales desde asuntos de orden estructural y no que emerjan desde posiciones de control y/o castigo.

Para finalizar este artículo, se considera que la buena práctica nombrada como replicabilidad y sostenibilidad permitirá construir bases sólidas que estructuren acciones ciudadanas responsables y generadoras de una cultura de la legalidad.

\section{Referencias}

Bauman, Z. (2005). Amor líquido: acerca de la fragilidad de los vínculos humanos. México: Fondo de Cultura Económica.

Berroeta, H. y Rodríguez, M. (2010). Una experiencia de participación comunitaria de regeneración del espacio público. Revista electrónica de psicología política 8(22), 1-26.

Cunill, N. (2010). Las políticas con enfoque de derechos y su incidencia en la institucionalidad pública. Revista del CLAD Reforma y Democracia, 46, 41-72.

Figueroa, O. y Orellana, A. (2007). Transantiago: gobernabilidad e institucionalidad. Revista Eure, 33(100), 165-171.

Galeano, M. (2013). Diseño de proyectos en la investigación cualitativa. Medellín: Fondo Editorial Universidad Eafit.

Gómez, Y., Usma, L., Zapata, L., Echavarría, R. y Giraldo, L. (2017). El control integral a las rentas ilícitas: Una experiencia de buenas prácticas en el Departamento de Antioquia. Medellín: Tecnológico de Antioquia Secretaría de Hacienda Gobernación de Antioquia. 


\section{Contexto zapata L. Lysma L}

Gumucio, A. (2004). El cuarto mosquetero: La comunicación para el cambio social. Investigación y desarrollo, 12(1), 2-23.

Incorporación, inclusión e integración. (07 de abril de 2009). Dinámica social y comunitaria. [Artículo en un blog]. Recuperado de http:// incorporacionintegracioneinclusion.blogspot.com/2009/04/posturasteoricas-sobre-dinamica-social.html

Martínez, R., Palma, A., Flores, L. y Paz, M. (2013). El impacto económico de las politicas sociales. Santiago de Chile: Naciones Unidas. Recuperado de https://repositorio.cepal.org/bitstream/handle/11362/4070/1/ S2013075_es.pdf

Montero, M. (2007). Introducción a la psicología comunitaria. Buenos Aires: Paidós.

Nagy, J. (s. f.). Estrategias para el cambio y mejora comunitaria: Una visión general. [Internet]. Caja de Herramientas Comunitarias. Centro para la Salud y Desarrollo Comunitario, Universidad de Kansas. Recuperado de https://ctb.ku.edu/es/tabla-de-contenidos/valoracion/promocion-estrategias/vision-general/principal

Piedra, J. (2017). Aproximación a la mediación comunitaria. Retos y desafíos. Revista de Mediación, 10(1), 1-7.

Polaski, S. (2003). Empleo, salarios e ingreso del grupo familiar. En J. Audley (Ed.), La promesa y la realidad del TLCAN (pp.11-40). México: Carnegie Endowment.

Revista Semana. (2000). Trabajo, crecimiento y desarrollo económico. Recuperado de https:/www.semana.com/nacion/articulo/trabajocrecimiento-desarrollo-economico/41919-3

Rojas, I. (2011). Hermenéutica para las técnicas cualitativas de investigación en ciencias sociales: una propuesta. Espacios públicos, 14(31), 176-189.

Téllez, E. (2010). El sentido del tejido social en la construcción de comunidad. Polisemia, 10, 9-23.

Usma, L. y Zapata, L. (2017). La cultura de la legalidad como un ejercicio de buenas prácticas en el Departamento de Antioquia. En-Contexto, 5(7), 255-275. 
Usma, L. y Zapata, L. (2018). La Cultura de la legalidad en las rentas del Departamento de Antioquia como un ejercicio de buenas prácticas desde las acciones comunitarias. En Y. Gómez (Ed.), Observatorio Público. Experiencias, tendencias y desafios. Líneas de investigación para explorar los campos de las Ciencias Administrativas y de Gestión, Económicas y Contables (pp. 430-449). Medellín: Publicar-T.

\section{Para citar este artículo:}

Zapata, L. y Usma, L. (2019). Los fraudes en las rentas departamentales: una mirada desde la institucionalidad a las dimensiones social, económica, legal y normativa. En-Contexto, 7(11), 213-229. 